Journal of Animal and Veterinary Advances 11 (10): 1656-1665, 2012

ISSN: $1680-5593$

(C) Medwell Journals, 2012

\title{
Cloning and Characterization of Oviduct Tubular Gland Cell Tissue-Specific Promoters of White Leghorn Chicken
}

\author{
${ }^{1,2}$ Jenn-Fa Liou, ${ }^{1,2}$ Chang-Hsin Chan, ${ }^{2}$ Jen-Wen Shiau, ${ }^{2}$ Jui-Jane Tailiu, ${ }^{1}$ Ming-Cheng Chang, \\ ${ }^{1,2,3}$ Lih-Ren Chen and ${ }^{3}$ Chein Tai \\ ${ }^{1}$ Institute of Biotechnology, National Chung Kung University, 701 Tainan, Taiwan, Republic of China \\ ${ }^{2}$ Physiology Division, Livestock Research Institute, COA-LRI, 712 Tainan, Taiwan, Republic of China \\ ${ }^{3}$ Southern Taiwan University, 710 Tainan, Taiwan, Republic of China
}

\begin{abstract}
Lactoferrin belongs to the transferrin family, the major function of which is in regulating the iron concentration in the blood stream and gastrointestinal tract. Lactoferrin has a remarkable antibacterial effect and plays an important role in the regulation of immune response. In a previous study, a full-length cDNA of $2133 \mathrm{bp}$ from Macaca cyclopis lactoferrin gene $(m L F)$ was cloned and sequenced. An effective promoter that can specifically drive the egg genes is necessary to establish a transgenic chicken line that can overexpress $\mathrm{mLF}$ in its eggs in addition to the viral promoters under development. Conalbumin and ovalbumin are the major proteins in egg white. The promoter regions of these two genes were cloned from the chromosomes of white Leghorn chicken in this study. This study uses fluorescence and luciferase assays to test the activities and tissue specificity of these two genes in primary cell cultures of white Leghorn chicken. The results show that the two promoters did not function in chicken muscle cells and fibroblast cells. However, the conalbumin promoter was active in chicken hepatocytes and oviduct tubular gland cells. By contrast, the ovalbumin promoter was active only in chicken primary oviduct tubular gland cells. Both promoters were regulated by a mixture of steroid hormones. The two promoters were also used to drive $\mathrm{mLF}$ expression in chicken primary hepatocytes and oviduct tubular gland cells. The results show that the ovalbumin and conalbumin promoters cloned in this study functioned in the chicken primary cell culture level. In the future, promoters will be further studied or reconstructed for gene transfer application in chicken.
\end{abstract}

Key words: Lactoferrin, ovalbumin, conalbumin, promoter, tissue specificity, Taiwan

\section{INTRODUCTION}

Lactoferrin (LF) is an iron-binding protein that is closely related to the plasma iron-transport protein transferrin. Lactoferrin is a single-chain glycoprotein with a molecular weight of approximately $80 \mathrm{kDa}$. Lactoferrin is folded into two lobes that show sequence homology with each other and each lobe can reversibly bind one ferric ion along with a synergistic anion, usually bicarbonate. In these respects it closely resembles transferrin although, its affinity for iron is relatively higher, allowing iron to be retained at lower $\mathrm{pH}$ values. Lactoferrin is also extremely basic with a $\mathrm{pI}$ of $8-9$, probably caused by a unique basic region in the N-terminal region of the molecule that is not found in transferrin. A wide spectrum of functions is ascribed to lactoferrin. These functions range from a role in the control of iron availability to immune modulation. Lactoferrin is highly conserved among human, mouse, bovine and porcine species. In a previous study, a fulllength cDNA of $2133 \mathrm{bp}$ from a Macaca cyclopis
Lactoferrin gene $(m L F)$ was cloned and sequenced. The nucleotide sequence of $\mathrm{mLF}$ shows high identity (93\%) to that of the human Lactoferrin gene $(h L F)$.

Like tissue culture and mammary gland bioreactors, the avian oviduct can also potentially serve as a bioreactor. The egg white of the hen consists of several layers secreted upon the yolk during its passage through the oviduct. After ovulation or release of the yolk from the ovary, the oocyte passes into the infundibulum of the oviduct, where it is fertilized if sperm are present. It then moves into the magnum of the oviduct which is lined with tubular gland cells. These cells secrete the egg white proteins including ovalbumin, lysozyme, ovomucoid, conalbumin and ovomucin into the lumen of the magnum where they are deposited onto the avian embryo and yolk.

Steroid hormones such as estrogen, progestin, glucocorticoids and androgens regulate the transcription of the two egg white proteins conalbumin (or ovotransferrin) and ovalbumin in chicken oviduct tubular gland cells (Cochet et al., 1979; Dierich et al.,

Corresponding Author: Chein Tai, Southern Taiwan University, Yungkang District, 710 Tainan, Taiwan, Republic of China 
1987; Lee et al., 1978; Palmiter, 1972; Perrin et al., 1979; Sanders and McKnight, 1985). In the absence of steroid hormones, a basal level of transcription of the conalbumin gene exists whereas transcription of the ovalbumin gene is undetectable (Hager et al., 1980; Hynes et al., 1979; Mulvihill and Palmiter, 1977; O'Malley et al., 1979). In chicken parenchymal liver cells, serum iron levels regulate the transcription of the conalbumin gene (McKnight et al., 1980a). This process is substantially less sensitive to steroid hormones than conalbumin transcription in the oviduct (Lee et al., 1978; McKnight et al., 1980b). In contrast, these cells never express the ovalbumin gene, despite the presence of functional oestrogen receptor molecules which allow estrogen induction of vitellogenin II gene transcription (Deeley et al., 1977; Jost et al., 1978; Wilks et al., 1981, 1982).

Changes in the rates of ovalbumin and conalbumin gene transcription and mRNA levels were monitored during an entire cycle of estrogen withdrawal and restimulation. The mechanisms by which steroid hormones mediate mRNA transcription and stability are generally assumed to involve transcriptional regulation mediated by nuclear steroid receptors that bind to specific sites near the genes they regulate (Palmiter et al., 1981). Conalbumin gene transcription is directly proportional to nuclear receptor levels whereas ovalbumin gene transcription is related to receptor levels in a manner that suggests cooperative interactions among receptors.

Ovalbumin gene expression is up-regulated 200 fold after estrogen administration in vivo. This is caused by a 20 fold increase in the transcription of the ovalbumin gene (Sanders and McKnight, 1988) coupled with a 10 fold increase in mRNA stability (Arao et al., 1996). Estrogen induction of the ovalbumin gene requires two cis-acting regulatory elements in the 5'-flanking region (Sanders and McKnight, 1988), the Steroid Dependent Regulatory Element (SDRE) which spans from -892-793 (Dean et al., 1998; Sanders and McKnight, 1988; Schweers et al., 1990) and the Negative Regulatory Element (NRE) which spans from 308-88 (Dillner and Sanders, 2002; Haecker et al., 1995; Sanders and McKnight, 1988; Sensenbaugh and Sanders, 1999). Furthermore in vivo genomic footprinting has identified three protein-protein complexes that bind to the SDRE after estrogen administration. Their complexes are called the chicken inducible regulatory proteins I-III (Chirp-I-III) (Dean et al., 1996, 1998; Schweers et al., 1990). The correlation between hypersensitivity and transcription was determined for each region in the chick oviduct where expression of the ovalbumin gene can be controlled by steroid hormones. Four DNase I-hypersensitive regions (I-IV) are centered approximately $0.15,0.80,3.2$ and $6.0 \mathrm{~kb}$ upstream from the
mRNA cap site in hen oviducts (Kaye et al., 1984). Ovalbumin mRNA levels are high in stimulated chicks where hypersensitive regions are present and drop to very low levels in unstimulated chicks where hypersensitivity is absent (Kaye et al., 1986).

This study shows that the ovalbumin and conalbumin promoters do not function in chicken fibroblasts, muscle cells and hepatocytes. The exception is the conalbumin promoter which shows basal level activity in primary cultured liver cells. However, both promoters are active in primary cultured chicken oviduct tubular gland cells. This reveals that conalbumin and ovalbumin promoters are not regulated by steroid hormones but show tissue specificity. Based on these characteristics of the conalbumin and ovalbumin promoters, we established a transgenic chicken line that can overexpress $\mathrm{mLF}$ protein driving by the conalbumin and ovalbumin promoters. Use of the conalbumin and ovalbumin promoters can avoid the biological dangers of virus promoters.

\section{MATERIALS AND METHODS}

Primary cultured cells: Chick embryo liver cells were prepared from 18 days old chicken embryos with minor modifications. After collagenase treatment, the cells were filtered through a nylon sieve (48 $\mu \mathrm{m}$ mesh) before centrifugation. The plated cells were incubated in Petri dishes for several hours in DME medium supplemented with $10 \%$ FBS and then Maintained in MEM medium containing insulin $\left(1 \mathrm{mg} \mathrm{mL}^{-1}\right)$ and antibiotics.

Primary cultured oviduct cells were prepared as follows. Oviduct magna were removed from 3 months old chickens. The oviducts were washed 3 times in sterile Phosphate Saline Buffer (PBS), minced with scissors in PBS and trypsinized gently $(0.5 \%$ trypsin in PBS-EDTA $1 \mathrm{mM}$ ) for $5 \mathrm{~min}$ at $37^{\circ} \mathrm{C}$. The supernatant was decanted and made $5 \%$ in FBS to inhibit trypsin action. The trypsinization of the pellets was repeated three or four times. All supernatants were pooled and filtered through gauze. The filtered cells were pelleted, resuspended in fresh DME medium supplemented with 10, FBS, 1\% L-glutamine, $1 \mathrm{mg} \mathrm{L}^{-1}$ insulin and cultivated in Petri dishes at $37^{\circ} \mathrm{C}$ in $95 \%$ air and $5 \% \mathrm{CO}_{2}$.

Plasmids construction: The fluorescence experiment in this study used Enhanced Yellow-green Fluorescent Protein (EYFP) expressed from the pEYFP-Nuc vector as the reporter. $\mathrm{pEYFP-cona}$ and $\mathrm{pEYFP-oval}$ were constructed by replacing the CMV promoter of the pEYFP-Nuc plasmid with the conalbumin and ovalbumin promoters, respectively. 
For luciferase assay, pGL3-Cona and pGL3-Oval were constructed by inserting the conalbumin and ovalbumin promoters into the Kpn I-Bgl II sites and Kpn I-Xho I sites, respectively, upstream of the luciferase gene.

pEGFP-cona-mLF and pEGFP-oval-mLF were used in the Western blotting experiment. To synthesize pEGFP$\mathrm{mLF}, \mathrm{mLF}$ was amplified by PCR using the pGEM-T-mLF as a template and primers containing cloning sites (5'CCGCTCGAGCGGCCGCCACCATGAAGCTTGTCTTC CTCG-3' and 5'-CGGGATCCCGCTTC-TGGAGGAATGCACAGG-3') and cloned into the Xho I-BamH I sites of the $\mathrm{pEGFP-N1}$ plasmid. The CMV promoter of the $\mathrm{pEGFP-}$ $\mathrm{N} 1$ plasmid was then replaced with the conalbumin and ovalbumin promoters to produce pEGFP-cona-mLF and pEGFP-oval-mLF plasmids.

Cell culture and transfection: Primary cultured cells were cultured in DMEM medium supplemented with 10\% FBS at $37^{\circ} \mathrm{C}$ in 95 air and $5 \% \mathrm{CO}_{2}$. When a confluent monolayer formed, the cells were trypsinized, washed and resuspended in fresh DMEM medium. Approximately, $5 \times 10^{5}$ cells were mixed with $15 \mu$ g plasmids in a $0.2 \mathrm{~cm}$ pulser cuvette. Transfection was performed using the ELECTRO CELL MANPULATORECM ${ }^{\circledR} 2001$ (BTX) with a pulse length of 10 (1-99), voltage or $150 \mathrm{~V}$ and one pulse. Cells were cultured at $37^{\circ} \mathrm{C}$ after electroporation.

For the fluorescence experiments, the green-yellow fluorescence was observed by fluorescence microscopy $48 \mathrm{~h}$ post-transfection.

Luciferase assay: About $1 \times 10^{6}$ cells were seeded in a $10 \mathrm{~cm}$ tissue culture dish and subsequently transfecting with $15 \mu \mathrm{g}$ of luciferase reporter plasmids and $15 \mu \mathrm{g}$ of pSV- $\beta$-galactosidase control plasmid (Promega) using electroporation. The cells were harvested $48 \mathrm{~h}$ after transfection. Transcriptional activity was measured with a luminometer. Conalbumin and ovalbumin promoter plasmids (pGL3-cona, pGL3-oval) were constructed by replacing Simian Virus 40 (SV40) promoters with conalbumin and ovalbumin promoters, respectively. The SV40 promoter (pGL3-control) was used as a positive control. Plasmid pSV- $\beta$-Galactosidase directing $\beta$ galactosidase expression from the SV40 promoter was used and $\beta$-galactosidase assay was performed with the same cell extracts for normalization of transfection efficiency. All of the data shown in this study were obtained from at least three independent experiments.

Western blot and ELISA analysis: The expression of $\mathrm{mLF}$-GFP recombinant protein was detected by Western blot analysis and quantified by ELISA.
For Western blotting, samples prepared from cell lysates were separated by 10\% SDS-PAGE. Following electrophoresis, proteins were transferred to a nitrocellulose membrane (Millipore) with a semidry horizontal Apparatus (AMMS). The human lactoferrin antibody was used as the primary antibody and Horseradish Peroxidase (HRP) conjugated secondary antibodies were used as the secondary antibody. The molecular mass of each protein was determined using a prestained protein ladder.

For ELISA analysis, the first and second antibodies were rabbit anti-hLF antibody (Sigma) and HRPconjugated mouse anti-rabbit IgG, respectively. Absorbance was read at $410 \mathrm{~nm}$ with a microplate reader. The expression level was calculated according to the standard curve derived from a serial dilution of the yeastexpressed $\mathrm{mLF}$ recombinant protein (from previous research). Plates were coated overnight with yeastexpressed $\mathrm{mLF}$ recombinant protein diluted with $0.1 \mathrm{~mol} \mathrm{~L}^{-1} \mathrm{NaHCO}_{3}$. Diluted mLF samples were then applied to these wells in duplicates and $\mathrm{hLF}$ antibody was detected with HRP-conjugated antirabbit IgG.

Statistical analysis: Student's t test was performed for statistical evaluation of the results. This study presents results as the arithmetic mean with the variance of the mean (mean $\pm \mathrm{SE})$.

\section{RESULTS AND DISCUSSION}

The conalbumin and ovalbumin promoter regions are active in primary cultured chicken oviduct tubular gland cells.

The 5'-flanking regions of conalbumin and ovalbumin genes were cloned from genomic DNA of white Leghorn chicken blood. To check the cloned 5'-flanking regions, the PCR products were sequenced and then aligned with the gene bank sequence (Fig. 1).

To confirm the 5'-flanking regions of conalbumin and ovalbumin genes, pEYFP-cona and pEYFP-oval recombinant plasmids were microinjected into primary cultured chicken oviduct tubular gland cells in the fluorescence experiment and pGL3-cona and pGL3-oval recombinant plasmids were used for the luciferase assay.

Figure 2a shows the different promoter activities in different primary cultured cell types. After transfecting control vectors $\mathrm{pEYFP-Nuc,} \mathrm{pEYFP-cona} \mathrm{and} \mathrm{pEYFP-}$ oval recombinant plasmids by electroporation, fluorescence microscopy revealed enhanced yellow-green fluorescent protein. This shows that conalbumin and ovalbumin promoters could drive the enhanced yellow- 
(a)

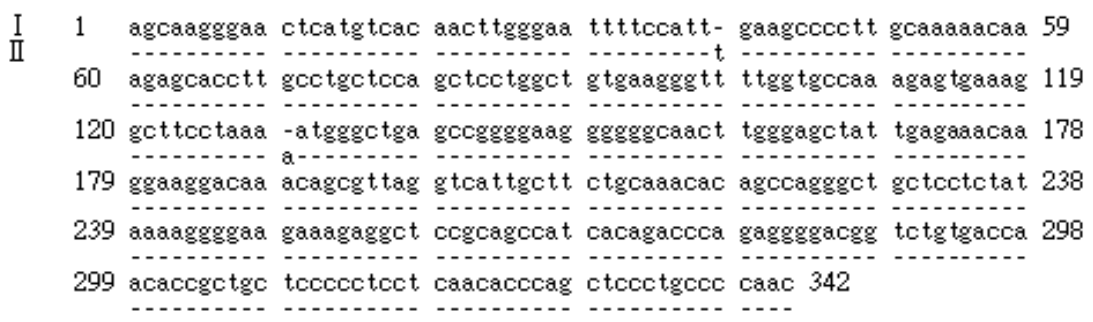

(b)

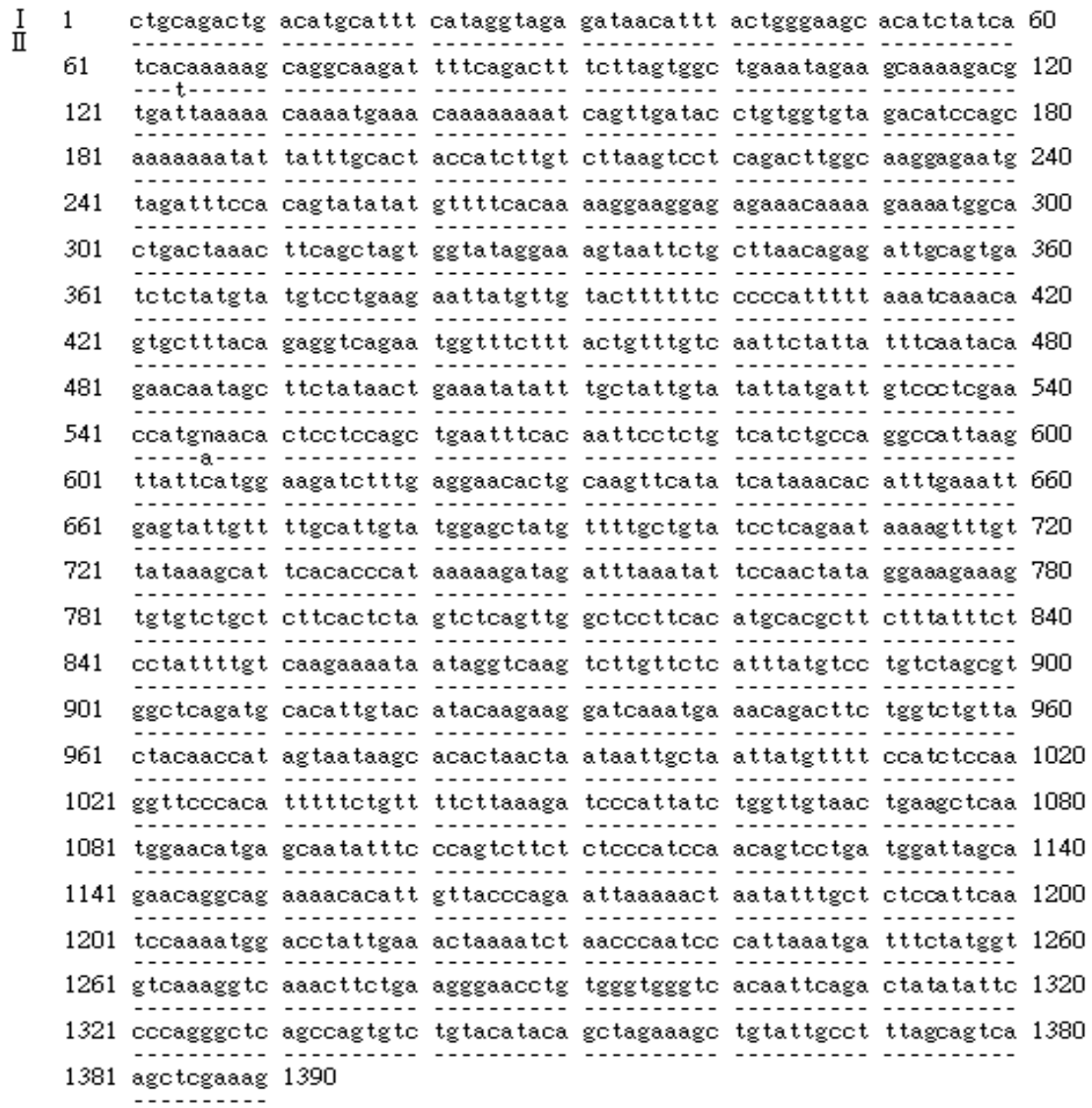

Fig. 1: a) Alignment of conalbumin and b) ovalbumin. I is obtained from the gene bank. II is the sequence we cloned. Dash means the nucleotide of II is identical to the nucleotide of I

green fluorescent protein expression in primary cultured oviduct tubular gland cells (Fig. 2a-I). In contrast, these two promoters were inactive in primary cultured fibroblast cells and primary cultured muscle cells (Fig. 2a-II, III). It exhibits yellow-green slightly in the field of the primary cultured embryonic liver cell transfected the pEYFP-cona (Fig. 2a-IV) but not pEYFP-oval.

A luciferase assay was used to confirm and quantify the promoter activity. The pGL3-control plasmid was transfected as a positive control and pGL3-basic was used as a negative control. We first lysed the primary cells to release luciferase and then measured its activity. The quantification of conalbumin and ovalbumin promoter activities in different cell types of primary cultured cell showed that the conalbumin promoter has similar activities in both primary cultured oviduct gland cells and liver cells. However, the ovalbumin promoter is active only in primary cultured oviduct gland cells. In the other primary cultured cells including fibroblast cells and muscle cells, neither the conalbumin promoter nor the 

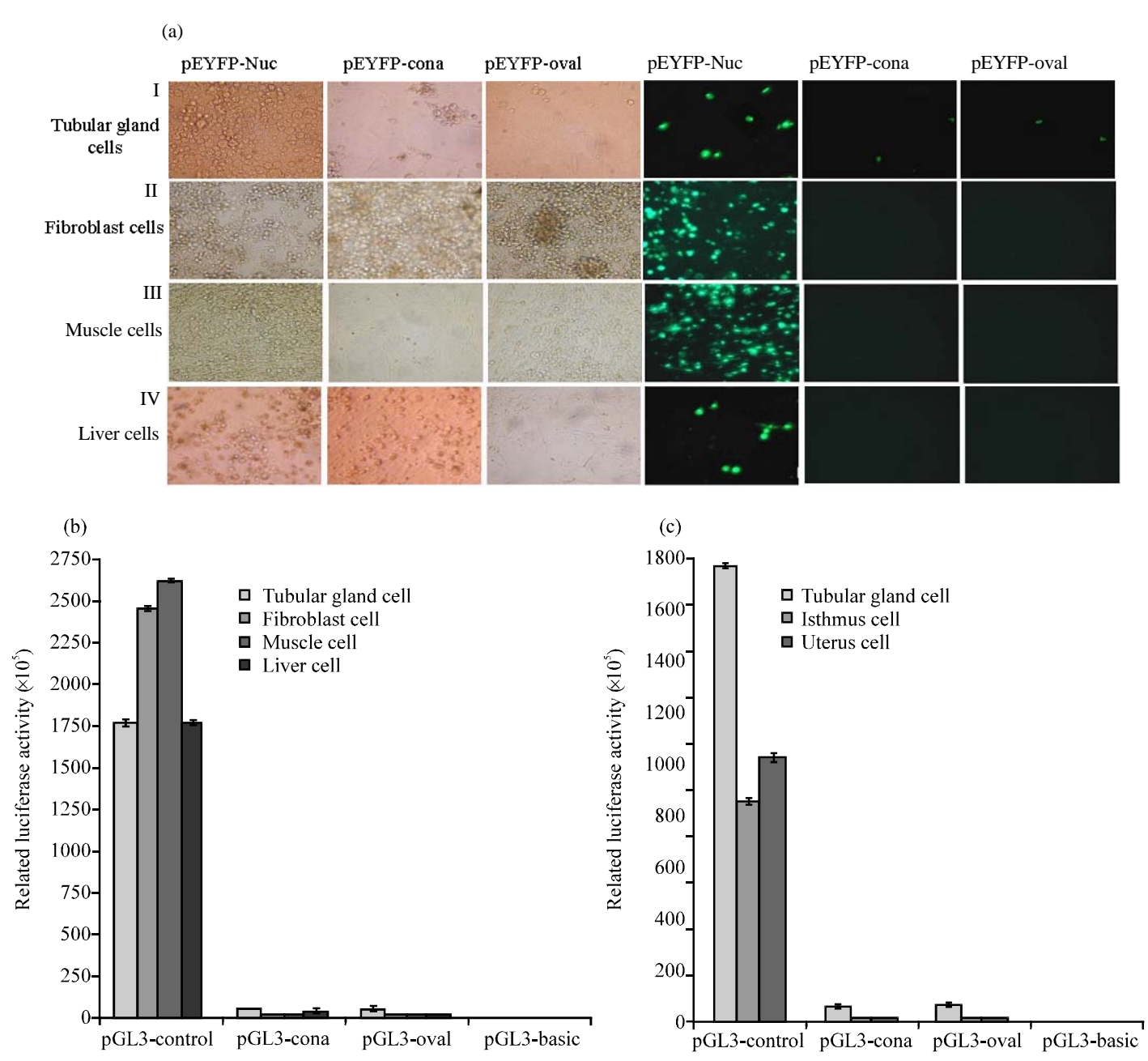

Fig. 2: a) Analysis of conalbumin and ovalbumin promoter activities in different primary cultured cells; $b$, c) observation of EYFP. Primary cultured cells were injected with the EYFP constructs as described in methods. About 2 days later expression of the reporter was visualized in primary cultured cells using a fluorescent microscope. Expressing embryos were photographed using a digital camera, Luciferase reporter assay for conalbumin and ovalbumin promoter activities. The Luciferase constructs containing conalbumin (pGL3-cona) or ovalbumin (pGL3-oval) promoters were transfected into different cell types of laying hens including; b) tubular gland cell, liver cell, fibroblast cell and muscle cell; c) or different segments of oviduct including tubular gland cell, isthmus cell and uterus cell. The cells were harvested $48 \mathrm{~h}$ after transfection for luciferase activity assays. The relative luciferase activities were normalized separately by using $\beta$-galactosidase values measured in the same cell extracts as the internal control. Data are represented as mean $\pm \mathrm{SD}$ of triplicate wells from three independent experiments

ovalbumin promoter was active (Fig. 2b). The synthesis of egg white proteins in the magnum including ovalbumin and conalbumin was significantly higher when an ovum was present than in any other segments such as isthmus and uterus (Muramatsu et al., 1991, 1994). To test this, we transfected pGL3-cona and pGL3-oval recombinant plasmids into primary cultured isthmus and uterus cells and measured luciferase activity $48 \mathrm{~h}$ after electroporation. The results demonstrated that the promoter activities of both conalbumin and ovalbumin promoters are higher in the primary cultured oviduct tubular magnum gland cell than in isthmus or uterus cells (Fig. 2c).

Combined, the 5'-flanking regions of the cloned conalbumin and ovalbumin reacted in a tissue-specific fashion. The activity of the ovalbumin and conalbumin promoter regions is controlled by steroid hormones in primary cultured chicken oviduct tubular gland cells but not in other cell types. The chicken oviduct system has 
contributed extensively to the knowledge of the hormonal regulation of gene expression. Estrogen induces several egg white proteins including ovalbumin and conalbumin (Kohler et al., 1969; O'Malley and McGuire, 1968; O'Malley et al., 1967). Characterization in vivo (Oka and Schimke 1969a, b; Palmiter, 1971; Palmiter and Haines, 1973; Palmiter and Wrenn, 1971) and in vitro using oviduct explant cultures (Compere et al., 1981; Hager et al., 1980; McKnight, 1978) has revealed that progestins androgens and glucocorticoids can also regulate the genes for these proteins if the chicks are estrogen treated. To test whether the cloned conalbumin and ovalbumin promoters reflect on steroid hormone treatment, all conalbumin and ovalbumin promoter recombinants were expressed in microinjected primary cultured oviduct tubular gland cells containing 10\% FBS, with oestradiol, progesterone and glucocorticoids at concentrations of $\sim 10^{-11}$ to $10^{-10} \mathrm{M}$. Unlike primary cultured oviduct tubular gland cells without hormone treatment, Fig. 3a shows higher EYFP expression.
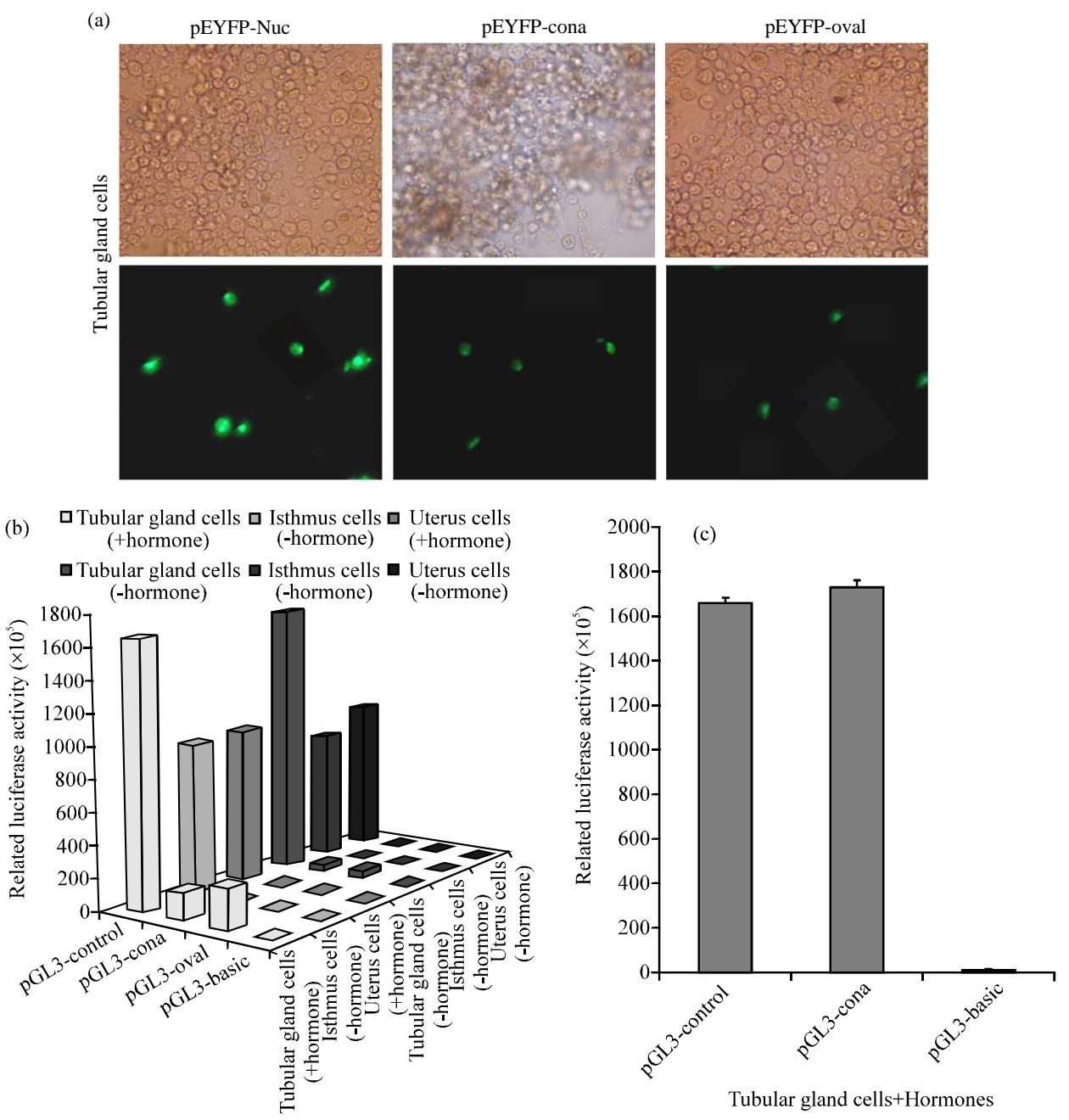

Fig. 3: a) The effect of steroid hormones on conalbumin and ovalbumin promoter activities in different primary cultured cells and b, c) observation of EYFP in primary cultured tubular gland cell treated with steroid hormones $\left(10^{-10} \mathrm{M}\right)$. Primary cultured tubular gland cell was injected with the EYFP constructs as described in methods. About 2 days later the expression of the reporter was visualized in primary cultured cells using a fluorescent microscope. Expressing embryos were photographed using a digital camera, the results of luciferase assay. The cell-lysated protein samples were freshly prepared from tubular gland cells isthmus cells and uterus cells treated with and without steroid hormones $\left(10^{-10} \mathrm{M}\right)$. The cells were harvested $48 \mathrm{~h}$ after transfection for luciferase activity assays. The relative luciferase activities were normalized separately by using $\beta$-galactosidase values measured in the same cell extracts as the internal control. Data are represented as mean $\pm \mathrm{SD}$ of triplicate wells from three independent experiments 
Whether treating the hormones affects different segments of the oviduct, we transfected GL3-cona and pGL3-oval into primary cultured tubular gland cells, isthmus cells and uterus cells maintained in DME medium containing 10\% FBS with and without oestradiol, progesterone and glucocorticoids at concentrations of $\sim 10^{-11}$ to $10^{-10} \mathrm{M}$ (Fig. 3b). Luciferase activity was then detected. These results reveal that both conalbumin and ovalbumin promoters are active in primary cultured tubular gland cells and show higher activity with hormone treatment.

In several instances, the activity of the conalbumin promoter was almost as high as positive control which contains SV40 promoter in primary cultured tubular gland cell of laying hens treated with steroid hormones (Fig. 3c).

These results confirm that steroid hormones upregulate the activity of conalbumin and ovalbumin promoters.

The expression of the $\mathrm{mLF}$ is under the control of conalbumin and ovalbumin promoter regions. The previous studies involved cloning the Macaca cyclopis lactoferrin gene ( $m L F$ gene). In the present study, to express the exogenous protein, $\mathrm{mLF}$, driven by the conalbumin or ovalbumin promoter, we constructed pEGFP-cona-mLF and pEGFP-oval-mLF recombinant plasmids. mLF-EGFP recombinant protein were detected by Western blotting $48 \mathrm{~h}$ after microinjection into chicken oviduct tubular gland cells or chicken embryonic liver cells maintained in DME medium containing 10\% FBS with oestradiol, progesterone and glucocorticoids at concentrations of $10^{-10} \mathrm{M}$. The Western blot results were consistent with observations of EYFP expression and analysis of luciferase assay. mLF-GFP recombinant protein was detectable in tubular gland cell lysates transfected with pEGFP-cona-mLF, pEGFP-oval-mLF and the positive control pEGFP-mLF (Fig. 4a). However in primary cultured liver cells, the results only detected conalbumin promoter and CMV promoter-driven mLF-GFP recombinant protein expression despite its weak expression (Fig. 4b).

The concentration of the pEGFP-mLF which served as a positive control containing the $\mathrm{CMV}$ promoter increased 10.5 fold compared with the lysate-only negative control whereas the concentration of pEGFPcona-mLF and pEGFP-oval-mLF increased 3.5 and 5.25 fold, respectively (Fig. 5). Thus, the expression of mLF-GFP recombinant protein was driven by both promoters of conalbumin and ovalbumin genes in primary cultured tubular gland cells.

A highly expressed oviduct promoter has not been developed. This is primarily because an established line of oviduct cells for promoter testing and an efficient
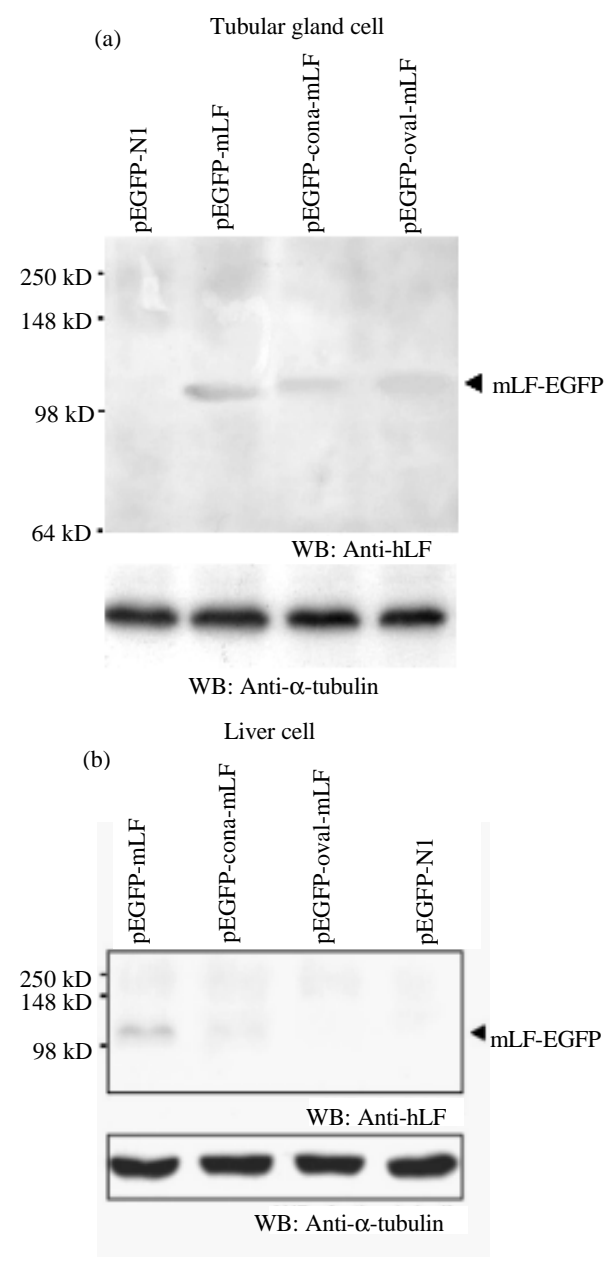

Fig. 4: Western blot analysis of mLF-GFP proteins from primary cultured tubular gland cells and liver cells. The cell-lysated protein samples were freshly prepared from; a) primary cultured tubular gland cells and b) liver cells treated with steroid hormones including $\beta$-estradiol, progesterone and corticosterone at the concentration of $10^{-10} \mathrm{M}$ and separated on 10\% SDS-PAGE (200 mg proteins/lane) followed by transferring to the nitrocellulose membranes. The transferred membranes were incubated with the anti-hLF antibody. Anti- $\alpha$-tubulin antibody against tubulin was used for internal control (bottom lanes). Only the vector pEGFP-N1 was used as the negative control as well as the pEGFP-coan-mLF and pEGFP-oval-mLF containing conalbumin and ovalbumin promoters, respectively and exogenous genes and $\mathrm{mLF}$ were test samples. The positive control was a CMV-driven construct, pEGFP-mLF. The experiments were repeated twice with similar results 


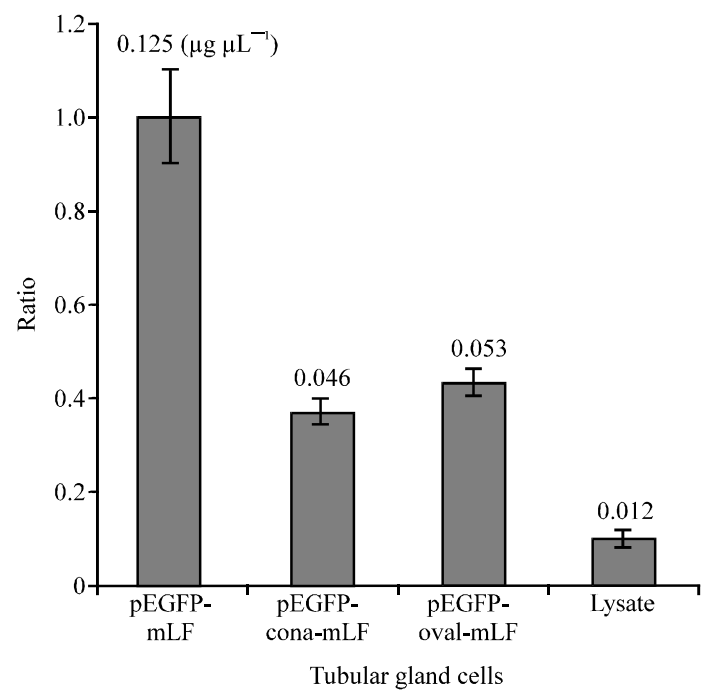

Fig. 5: Ratio of the mLF-GFP recombinant protein expression by ELISA analysis. The three constructs shown in Fig. 4 were analyzed for quantified protein expression. Data are based on three experiments and presented as means $\pm \mathrm{SD}$

method for producing transgenic chickens has been scant. However, the ovalbumin promoter has been analyzed extensively in explant cultures of immature chick oviducts stimulated to grow and differentiate by steroid hormones (Dean et al., 1996; Haecker et al., 1995; Sanders and McKnight, 1988; Schweers and Sanders, 1991). The experiments in this study used the primary cultured oviduct tubular gland cells of 3 months old hens and maintained them for eight generations.

To analyze the ovalbumin promoter, four hormonally induced DNase I-hypersensitive sites were generated upstream of the transcription start site (Kaye et al., 1984, 1986). These sites most often represent regions in chromatin structurally rearranged by bound transcription factors enabling DNA access for digestion. Proximal sites I (negative regulatory element, NRE) (Haecker et al., 1995; Sanders and McKnight, 1988; Sensenbaugh and Sanders, 1999) and II (steroid-dependent response element, SDRE) (Dean et al., 1998; Sanders and McKnight, 1988; Schweers et al., 1990) are necessary for steroid-mediated expression in chick oviduct explant cultures (Kato et al., 1992). The SDRE binds a steroid-inducible protein Chirp-1 (Dean et al., 1998, 1996; Schweers et al., 1990) and is thought to relieve transcriptional repression at the NRE. Hypersensitive site III contains half-palindromic estrogen response elements that can mediate hormone induction in transfected Hela cells (Kato et al., 1992) whereas the function of distal site IV is unknown.
The two proximal sites lie within $850 \mathrm{bp}$ of the transcription start site which is within the ovalbumin promoter region cloned in this study.

The hen has numerous advantages over other systems as a bioreactor. Unlike cows, goats and sheep, chickens have been raised for many generations as specific pathogen-free animals in biosecure facilities. Unlike milk biologics, human and animal vaccines produced in eggs have a long regulatory history. The short generation times and prolific rates of reproduction allow production to be upscaled rapidly. Unlike cell-based production systems which operate most efficiently in batch mode, chickens continuously produce eggs yearround, allowing efficient use of downstream facilities for purification and processing.

\section{CONCLUSION}

This study shows that the hen which has highthroughput and low-cost infrastructure is useful for producing exogenous protein such as $\mathrm{mLF}$.

\section{REFERENCES}

Arao, Y., N. Miyatake, E. Yamamoto, H. Usiku and A. Matsuura et al., 1996. Steroid hormones differentially induce transcription of the chicken ovalbumin gene, but stabilize the mRNA with the same half-life. J. Biochem., 120: 710-715.

Cochet, M., F. Perrin, F. Gannon, A. Krust and P. Chambon et al., 1979. Cloning of an almost fulllength chicken conalbumin double-stranded cDNA. Nucl. Acids Res., 6: 2435-2452.

Compere, S.J., G.S. McKnight and R.D. Palmiter, 1981. Androgens regulate ovomucoid and ovalbumin gene expression independently of estrogen. J. Biol. Chem., 256: 6341-6347.

Dean, D.M., P.S. Jones and M.M. Sanders, 1996. Regulation of the chicken ovalbumin gene by estrogen and corticosterone requires a novel DNA element that binds a labile protein, Chirp-1. Mol. Cell Biol., 16: 2015-2024.

Dean, D.M., R.R. Berger and M.M. Sanders, 1998. A winged-helix family member is involved in a steroid hormone-triggered regulatory circuit. Endocrinology, 139: 4967-4975.

Deeley, R.G., J.I. Gordon, A.T. Burns, K.P. Mullinix, M. Binastein and R.F. Goldberg, 1977. Primary activation of the vitellogenin gene in the rooster. J. Biol. Chem., 252: 8310-8319.

Dierich, A., M.P. Gaub, J.P. LePennec, D. Astinotti and P. Chambon, 1987. Cell-specificity of the chicken ovalbumin and conalbumin promoters. Embo J., 6: 2305-2312. 
Dillner, N.B. and M.M. Sanders, 2002. Upstream stimulatory factor (USF) is recruited into a steroid hormone-triggered regulatory circuit by the estrogeninducible transcription factor delta EF1. J. Biol. Chem., 277: 33890-33894.

Haecker, S.A., T. Muramatsu, K.R. Sensenbaugh and M.M. Sanders, 1995. Repression of the ovalbumin gene involves multiple negative elements including a ubiquitous transcriptional silencer. Mol. Endocrinol., 9: 1113-1126.

Hager, L.J., G.S. McKnight and R.D. Palmiter, 1980. Glucocorticoid induction of egg white J. Biol. Chem., 255: 7796-7800.

Hynes, N.E., B. Groner, A.E. Sippel, S. Jeep and T. Wurtz et al., 1979. Control of cellular content of chicken egg white protein specific RNA during estrogen dministration and withdrawal. Biochemistry, 18: $616-624$.

Jost, J.P., T. Ohno, S. Panyim and A.R. Schuerch, 1978. Appearance of vitellogenin mRNA sequences and rate of vitellogenin synthesis in chicken liver following primary and secondary stimulation by 17 beta-estradiol. Eur. J. Biochem., 84: 355-361.

Kato, S., L. Tora, J. Yamauchi, S. Masushige, M. Bellard and P. Chambon, 1992. A far upstream estrogen response element of the ovalbumin gene contains several half-palindromic 5'-TGACC-3' motifs acting synergistically. Cell, 68: 731-742.

Kaye, J.S., M. Bellard, G. Dretzen, F. Bellard and P. Chambon, 1984. A close association between sites of DNase I hypersensitivity and sites of enhanced cleavage by micrococcal nuclease in the 5 '-flanking region of the actively transcribed ovalbumin gene. Embo J., 3: 1137-1144.

Kaye, J.S., S. Pratt-Kaye, M. Bellard, G. Dretzen, F. Bellard and $\mathrm{P}$. Chambon, 1986. Steroid hormone dependence of four DNase I-hypersensitive regions located within the 7000-bp 5'-flanking segment of the ovalbumin gene. Embo J., 5: 277-285.

Kohler, P.O., P.M. Grimley and B.W. O'Malley, 1969. Estrogen-induced cytodifferentiation of the ovalbumin-secreting glands of the chick oviduct. J. Cell Biol., 40: 8-27.

Lee, D.C., G.S. McKnight and R.D. Palmiter, 1978. The action of estrogen and progesterone on the expression of the transferrin gene. A comparison of the response in chick liver and oviduct. J. Biol. Chem., 253: 3494-3503.

McKnight, G.S., 1978. The induction of ovalbumin and conalbumin mRNA by estrogen and progesterone in chick oviduct explant cultures. Cell, 14: 403-413.
McKnight, G.S., D.C. Lee and R.D. Palmiter, 1980 b. Transferrin gene expression. Regulation of mRNA transcription in chick liver by steroid hormones and iron deficiency. J. Biol. Chem., 255: 148-153.

McKnight, G.S., D.C. Lee, D. Hemmaplardh, C.A. Finch and R.D. Palmiter, 1980a. Transferrin gene expression. Effects of nutritional iron deficiency. J. Biol. Chem., 255: 144-147.

Mulvihill, E.R. and R.D. Palmiter, 1977. Relationship of nuclear estrogen receptor levels to induction of ovalbumin and conalbumin mRNA in chick oviduct. J. Biol. Chem., 252: 2060-2068.

Muramatsu, T., H. Hiramatsu and J. Okumura, 1994. Ovalbumin mRNA in the magnum of laying hens changes during egg formation cycle. Br. Poult Sci., 35: 457-461.

Muramatsu, T., K. Hiramoto and J. Okumura, 1991. Changes in ovalbumin and protein synthesis in vivo in the magnum of laying hens during the egg formation cycle. Comp. Biochem. Physiol. B., 99: 141-146.

O'Malley, B.W. and W.L. McGuire, 1968. Studies on the mechanism of estrogen-mediated tissue differentiation: Regulation of nuclear transcription and induction of new RNA species. Proc. Natl. Acad. Sci. USA., 60: 1527-1534.

O'Malley, B.W., D.R. Roop, E.C. Lai, J.L. Nordstrom and J.F. Catterall et al., 1979. The ovalbumin gene: organization, structure, transcription and regulation. Recent, Prog. Horm. Res., 35: 1-46.

O'Malley, B.W., W.L. McGuire and S.G. Korenman, 1967. Estrogen stimulation of synthesis of specific proteins and RNA polymerase activity in the immature chick oviduct. Biochim. Biophys. Acta, 145: 204-207.

Oka, T. and R.T. Schimke, 1969b. Interaction of estrogen and progesterone in chick oviduct development. Effects of estrogen and progesterone on tubular gland cell function. J. Cell Biol., 43: 123-137.

Oka, T. and R.T. Schimke, 1969a. Progesterone antagonism of estrogen-induced cytodifferentiation in chick oviduct. Science, 163: 83-85.

Palmiter, R.D. and J.T. Wrenn, 1971. Interaction of estrogen and progesterone in chick oviduct development. III. Tubular gland cell cytodifferentiation. J. Cell Biol., 50: 598-615.

Palmiter, R.D. and M.E. Haines, 1973. Regulation of protein synthesis in chick oviduct. IV, Role of testosterone. J. Biol. Chem., 248: 2107-2116.

Palmiter, R.D., 1971. Interaction of estrogen, progesterone and testosterone in the regulation of protein synthesis in chick oviduct. Biochemistry, 10: 4399-4403. 
Palmiter, R.D., 1972. Regulation of protein synthesis in chick oviduct. Independent regulation of ovalbumin, conalbumin, ovomucoid and lysozyme induction. J. Biol. Chem., 247: 6450-6461.

Palmiter, R.D., E.R. Mulvihill, J.H. Shepherd and G.S. McKnight, 1981. Steroid hormone regulation of ovalbumin and conalbumin gene transcription. A model based upon multiple regulatory sites and intermediary proteins. J. Biol. Chem., 256: 7910-7916.

Perrin, F., M. Cochet, P. Gerlinger, B. Cami, J.P. LePennec and P. Chambon, 1979. The chicken conalbumin gene: Studies of the organization of cloned DNAs. Nucl. Acids Res., 6: 2731-2748.

Sanders, M.M. and G.S. McKnight, 1985. Chicken egg white genes: Multihormonal regulation in a primary cell culture system. Endocrinology, 116: 398-405.

Sanders, M.M. and G.S. McKnight, 1988. Positive and negative regulatory elements control the steroidresponsive ovalbumin promoter. Biochemistry, 27: $6550-6557$.
Schweers, L.A. and M.M. Sanders, 1991. A protein with a binding specificity similar to NF-kappa B binds to a steroid-dependent regulatory element in the ovalbumin gene. J. Biol. Chem., 266: 10490-10497.

Schweers, L.A., D.E. Frank, N.L. Weigel and M.M. Sanders, 1990. The steroid-dependent regulatory element in the ovalbumin gene does not function as a typical steroid response element. J. Biol. Chem., 265: 7590-7595.

Sensenbaugh, K.R. and M.M. Sanders, 1999. Multiple promoter elements including a novel repressor site modulate expression of the chick ovalbumin gene. DNA Cell Biol., 18: 147-156.

Wilks, A., A.C. Cato, P.J. Cozens, I.W. Mattaj and J.P. Jost, 1981. Isolation and fine structure organisation of an avian vitellogenin gene coding for the major estrogen-inducible mRNA. Genetics, 16: 249-259.

Wilks, A.F., P.J. Cozens, I.W. Mattaj and J.P. Jost, 1982. Estrogen induces a demethylation at the $5^{\prime}$ end region of the chicken vitellogenin gene. Proc. Natl. Acad. Sci. USA., 79: 4252-4255. 